Pacific Journal of Mathematics

THE GROUP CHARACTER AND SPLIT GROUP ALGEBRAS 


\title{
THE GROUP CHARACTER AND SPLIT GROUP ALGEBRAS
}

\section{GEORGE SZeto}

\begin{abstract}
G. J. Janusz defined a splitting ring $R$ for a group $G$ of order $n$ invertible in $R$. Then, the Brauer splitting theorem was given by $G$. Szeto which proves the existence of a finitely generated projective and separable splitting ring for $G$. Let $M$ be a $R G$-module and $R_{0}$ be a subring of $R$. Then we say that $M$ is realizable in $R_{0}$ if and only if there exists a $R_{0} G$ module $N$ such that $M \cong R \otimes_{R_{0}} N$ as left $R G$-modules. This paper gives a characterization of splitting rings in terms of the concept of realizability as in the field case. The other main results in this paper are the structure theorem for split group algebras and some properties of group characters.
\end{abstract}

Throughout this paper we assume that the ring $R$ is a commutative ring with no idempotents except 0 and 1 , that the group $G$ has order $n$ invertible in $R$, and that all $R G$-modules are unitary left $R G$-modules. We know that the order of $G, n$, is invertible in $R$ if and only if $R G$ is separable.

1. In this section we study splitting rings in two ways. That is, splitting rings can be characterized in terms of the concept of realizability and structure theorem for split group algebras will be given.

Proposition 1. Assume the ring $R$ has no idempotents except 0 and 1 , and $P$ is a finitely generated and projective $R$-module. Then $P$ is a faithful $R$-module.

Proof. Because $P$ is a finitely generated and projective $R$-module, $R=\alpha(P)+\operatorname{Tr}(P)$ where $\alpha(P)$ is the kernel of the operation of $R$ on $P$ and $\operatorname{Tr}(P)$ is the trace ideal of $P$ in $R$ ([3], Proposition A.3). Thus $\alpha(P)$ is a left direct summand of $R$ ([3], Th. A.2(d)). But $R$ has no idempotents except 0 and 1 so that $\alpha(P)=0$. Therefore $P$ is a faithful $R$-module.

Using the above proposition we can have the following definition given by G. J. Janusz.

Definition 1. A ring $R$ is a splitting ring for $G$ if the group algebra $R G$ is the direct sum of central separable $R$-algebras, each equivalent to $R$ in the Brauer group of $R$; that is, 


$$
R G \cong \bigoplus \sum_{i=1}^{s} \operatorname{Hom}_{R}\left(P_{i}, P_{i}\right)
$$

where $\left\{P_{i}\right\}$ are finitely generated and projective $R$-modules. The number of different conjugate classes in $G$ is equal to $s$ ([5], Definition $6)$.

Definition 2. Let $M$ be a $R G$-module and $R_{0}$ be a subring of $R$. Then we say that $M$ is realizable in $R_{0}$ if and only if there exists a $R_{0} G$-module $N$ such that $M \cong R \bigotimes_{R_{0}} N$ as left $R G$-modules.

THEOREM 2. If $R$ is strongly separable over $R_{0}$ and $R$ is a splitting ring for $G, R G \cong \bigoplus \sum_{i=1}^{s} \operatorname{Hom}_{R}\left(P_{i}, P_{i}\right)$; then $P_{i}$ is realizable in $R_{0}$ for all $i$ if and only if $R_{0}$ is a splitting ring for $G$.

Proof. If $R_{0}$ is a splitting ring for $G$, that is, if

$$
R_{0} G \cong \bigoplus \sum_{i=1}^{s} \operatorname{Hom}_{R_{0}}\left(P_{i}, P_{i}\right) \text {, }
$$

then $P_{i} \cong R_{0} \bigotimes_{R_{0}} P_{i}$. This means that $P_{i}$ is realizable in $R_{0}$ for all $i$.

Conversely, if $P_{i}$ is realizable in $R_{0}$ for all $i$, then there is $R_{0} G$ module $M_{i}$ such that $P_{i} \cong R \bigotimes_{R_{0}} M_{i}$ for all $i$. Since $R$ is a strongly separable $R_{0}$-algebra, $R_{0} \cdot 1$ is a $R_{0}$-direct summand of $R$. By the definition of a split group algebra, $P_{i}$ is a finitely generated and projective $R$-module for each $i$; so $M_{i}$ is a finitely generated and projective $R_{0}$-module for each $i$. In fact, because $R \cong\left(R_{0} \cdot 1 \oplus R_{0}^{\prime}\right)$ for some $R_{0}$ module $R_{0}^{\prime}$,

$$
P_{i} \cong\left(R_{0} \cdot 1 \oplus R_{0}^{\prime}\right) \bigotimes_{R_{0}} M_{i} \cong\left(R_{0} \cdot 1 \bigotimes_{R_{0}} M_{i}\right) \oplus\left(R_{0}^{\prime} \bigotimes_{R_{0}} M_{i}\right) .
$$

Thus $M_{i} \cong R_{0} \cdot 1 \bigotimes_{R_{0}} M_{i}$ is a $R_{0}$-direct summand of $P_{i}$. On the other hand, $P_{i}$ is finitely generated and projective over $R$ and $R$ is finitely generated and projective over $R_{0}$; so $P_{i}$ is finitely generated and projective over $R_{0}$. Therefore $M_{i}$ is a finitely generated and projective $R_{0}$-module. We then have

$$
\begin{aligned}
R G \cong \bigoplus \sum_{i=1}^{s} \operatorname{Hom}_{R}\left(P_{i}, P_{i}\right) & \cong \bigoplus \sum_{i=1}^{s} \operatorname{Hom}_{R}\left(R \bigotimes_{R_{0}} M_{i}, R \bigotimes_{R_{0}} M_{i}\right) \\
& \cong R \bigotimes_{R 0}\left(\bigoplus \sum_{i=1}^{s} \operatorname{Hom}_{R_{0}}\left(M_{i}, M_{i}\right)\right) .
\end{aligned}
$$

Noting that $M_{i}$ is a finitely generated projective and faithful $R_{0}$-module for each $i$ by Proposition 1, we have that $\operatorname{Hom}_{R_{0}}\left(M_{i}, M_{i}\right)$ is a central separable $R_{0}$-algebra with a unique central idempotent in $R_{0} G$ for each $i$ ([2], Proposition 5.1). Therefore $R_{0} G \cong \bigoplus \sum_{i=1}^{s} \operatorname{Hom}_{R_{0}}\left(M_{i}, M_{i}\right)$. This proves that $R_{0}$ is a splitting ring for $G$. 
We are going to discuss the structure of a split group algebra over some kinds of rings, in particular, over a Dedekind ring.

THEOREM 3. Let $P$ denote a finitely generated and projective $R$-module. (a) If $R$ is a Dedekind domain, then $\operatorname{Hom}_{R}(P, P)$ is free as a $R$-module. Consequently, a split group algebra is a free $R$ module. (b) If $R$ is a local ring or a semi-local ring or a principal ideal Dedekind domain, then $\operatorname{Hom}_{R}(P, P)$ is a matrix ring over $R$.

Proof. Because $P$ is a finitely generated and projective $R$-module, $\operatorname{Hom}_{R}(P, P) \cong P \otimes_{R} \operatorname{Hom}_{R}(P, R)$. Let the rank of $P$ be $k$. Then $P \cong$ $\bigoplus \sum_{i=1}^{k-1} R \oplus I, \sum_{i=1}^{k-1} R$ are $k-1$ copies of $R$ and $I$ is in the class group of $R$. By substitution,

$$
\begin{aligned}
& P \bigotimes_{R} \operatorname{Hom}_{R}(P, R) \cong\left(\bigoplus \sum_{i=1}^{k-1} R \oplus I\right) \bigotimes_{R} \operatorname{Hom}_{R}\left(\oplus \sum_{i=1}^{k-1} R \oplus I, R\right) \\
& \cong\left(\oplus \sum_{i=1}^{k-1} R \oplus I\right) \otimes_{R}\left(\oplus \sum_{i=1}^{k-1} \operatorname{Hom}_{R}(R, R) \oplus \operatorname{Hom}_{R}(I, R)\right) \\
& \cong\left(\bigoplus \sum_{i=1}^{k-1} R \oplus I\right) \otimes_{R}\left(\bigoplus \sum_{i=1}^{k-1} R \oplus I^{-1}\right) \\
& \cong\left(\oplus \sum_{i=1}^{(k-1)^{2}} R\right) \oplus\left(\oplus \sum_{i=1}^{k-1} R \bigotimes_{R} I^{-1}\right) \oplus\left(\oplus \sum_{i=1}^{k-1} R \bigotimes_{R} I\right) \oplus\left(I \bigotimes_{R} I^{-1}\right) \\
& \cong\left(\oplus^{(k-1)^{2}} R\right) \oplus\left(\oplus \sum_{i=1}^{k-1} I^{-1}\right) \oplus\left(\oplus \sum_{i=1}^{k-1} I\right) \oplus R \\
& \cong\left(\oplus \sum_{i=1}^{(k-1)^{2}+1} R\right) \oplus\left(\oplus \sum_{i=1}^{2 k-2} R\right) \\
& \cong\left(\oplus \sum_{i=1}^{k^{2}}\right) R \text {. This proves part (a). }
\end{aligned}
$$

For part (b), because $P$ is a free module of finite rank over each of these rings, $\operatorname{Hom}_{R}(P, P)$ is a matrix ring over $R$. For a local ring $R$, see Theorem 12 in Chapter 9 in [6]. For a semi-local ring $R$, see the remark on Theorem 3.6 in [2]. For a principal ideal Dedekind domain, see Exercises 22.5 and 56.6 in [4].

REMARK. There exist split group algebras over those rings in the above theorem from the proof of the Brauer splitting theorem ([8], Th. 2).

Theorem 4. Let $R$ denote a Dedekind domain, $P$ a finitely generated and projective $R$-module and $P(R)$ the class group of $R$. Then, for $P \cong \oplus \sum_{i=1}^{k-1} R \oplus J$, there is $I$ in $P(R)$ such that $I^{k}=J^{-1}$ where $k=\operatorname{rank}(P)$ and $J$ is in $P(R)$ if and only if $\operatorname{Hom}_{R}(P, P)$ is a matrix ring over $R$ of order $k$ by $k$. 
Proof. Because $\operatorname{Hom}_{R}(P, P)$ is a matrix ring over $R$ if and only if there exists $I$ in $P(R)$ such that $P \bigotimes_{R} I \cong \bigoplus \sum_{i=1}^{k} R$, a direct sum of $k$-copies of $R$ (Lemma 9, [7]). But $P \cong \oplus \sum_{i=1}^{k-1} R \oplus J$ for some

$$
\begin{aligned}
J \text { in } P(R) ; \text { so } & \left(\left(\oplus \sum_{i=1}^{k-1} R\right) \oplus J\right) \bigotimes_{R} I \cong \bigoplus \sum_{i=1}^{k} R, \\
& \left(\bigoplus \sum_{i=1}^{k-1} I\right) \oplus\left(J \bigotimes_{R} I\right) \cong \bigoplus \sum_{i=1}^{k} R, \\
& \left(\bigoplus \sum_{i=1}^{k-1} I\right) \oplus(J \cdot I) \cong \bigoplus \sum_{i=1}^{k} R
\end{aligned}
$$

where we use the fact that $J \bigotimes_{R} I \cong J \cdot I$. But

$$
\left(\bigoplus \sum_{i=1}^{k-1} I\right) \oplus(J \cdot I) \cong \bigoplus_{i=1}^{k-1} R \oplus I^{k} \cdot J ;
$$

then $I^{k} \cdot J=R$. So, if we can prove the fact that $J \bigotimes_{R} I \cong J \cdot I$, the theorem is proved. In fact, because $J \cdot I$ is in $P(R)$ and $J \cdot I$ is projective and finitely generated, the exact sequence

$$
0 \longrightarrow \operatorname{Ker}(\pi) \longrightarrow J \otimes_{R} I \stackrel{\pi}{\longrightarrow} J \cdot I \longrightarrow 0
$$

splits. Thus $J \bigotimes_{R} I \cong \operatorname{Ker}(\pi) \oplus J \cdot I$. Let $R_{M}$ denote the quotient ring with respect to a prime ideal $M$.

$$
R_{M} \otimes_{R}\left(J \bigotimes_{R} I\right) \cong R_{M} \bigotimes_{R} \operatorname{Ker}(\pi) \oplus R_{M} \otimes_{R}(J \cdot I),
$$

that is, $R_{M} \cong R_{M} \otimes_{R} \operatorname{Ker}(\pi) \oplus R_{M}$. Hence $R_{M} \otimes_{R} \operatorname{Ker}(\pi)=0$ for all prime ideals $M$. On the other hand, because $\operatorname{Ker}(\pi)$ is finitely generated, $\operatorname{Ker}(\pi)=0$ by Nakayama's lemma. This proves that $J \bigotimes_{R} I \cong$ $J \cdot I$. Therefore the theorem is completed.

CoROLlary 5. Keep the same notations as Theorem 4. If the rank of $P$ and the order of $J$ are relative prime, then $\operatorname{Hom}_{R}(P, P)$ is a matrix ring over $R$.

Proof. It suffices to prove that there exists $I$ in $P(R)$ such that $J^{-1}=I^{k}$ by Theorem 4. Consider the subgroup generated by $J^{k}$. Because $k$, the rank of $P$ and the order of $J$ are relative prime, this subgroup is the same as the subgroup generated by $J$. Hence $J=$ $J^{i k}$ for some $i$ from 1 to the order of $J$ minus 1. Thus $I=\left(J^{-1}\right)^{i}$ is what we want. In fact, $I^{k}=\left(J^{-1}\right)^{i k}=\left(J^{i k}\right)^{-1}=J^{-1}$.

Definition 3. The subgroup of $P(R), U$, is called the $R-Z$ group for a finitely generated and projective $R$-module $P$ if $U=\{I$ such that $I$ is in $P(R)$ and $I \cdot P=P\}$. (For this group see Theorem 14 and Theorem 15 in [7]). 
THeOREM 6. (a) Let $R$ be a Dedekind domain and $H=\{J$ such that $P \cong \bigoplus \sum_{i=1}^{k-1} R \oplus J$ and $\operatorname{Hom}_{R}(P, P)$ is a matrix ring over $R$ where $J$ is in $P(R)\}$. Then $H$ is a subgroup of $P(R)$. (b) Assume the $R-$ $Z$ group is equal to $P(R)$. Then, $P$ is a free $R$-module if and only if $\operatorname{Hom}_{R}(P, P)$ is a matrix ring over $R$.

Proof. For any $J^{\prime}$ and $J^{\prime \prime}$ in $H$, there are $I^{\prime}$ and $I^{\prime \prime}$ in $P(R)$ such that $J^{\prime} \cdot\left(I^{\prime}\right)^{k}=R$ and $J^{\prime \prime} \cdot\left(I^{\prime \prime}\right)^{k}=R$ by Theorem 4 . We then have $J^{\prime} \cdot J^{\prime \prime} \cdot\left(I^{\prime} \cdot I^{\prime \prime}\right)^{k}=\left(J^{\prime} \cdot I^{\prime k}\right)\left(J^{\prime \prime} \cdot I^{\prime \prime k}\right)=R$. Thus $J^{\prime} \cdot J^{\prime \prime}$ is in $H$. Also, for any $J$ in $H$, there is $I$ in $P(R)$ such that $J \cdot I^{k}=R$. We then have $J^{-1} \cdot\left(I^{k}\right)^{-1}=R$, that is, $J^{-1} \cdot\left(I^{-1}\right)^{k}=R$. Thus $J^{-1}$ is in $H$. Therefore $H$ is a subgroup of $P(R)$. This proves part (a).

For part (b), one way is clear. If $P$ is free, then $\operatorname{Hom}_{R}(P, P)$ is a matrix ring over $R$. Conversely, if $\operatorname{Hom}_{R}(P, P)$ is a matrix ring over $R, P \cong \oplus \sum_{i=1}^{k-1} R \oplus J$ with $J$ in $H$ by Theorem 4 . But the $R-Z$ group is equal to $P(R)$; then $I^{k}=R$ for all $I$ in $P(R)$. Thus $H=0$. Therefore $P$ is a free $R$-module.

REMARK. (a) Corollary 5 can be expressed in terms of the $R-Z$ group as following. If the exponent of the $R-Z$ group and the order of $J$ is relative prime, then $\operatorname{Hom}_{R}(P, P)$ is a matrix ring over $R$.

(b) Theorem 4, Corollary 5, and Theorem 6 tell us the structure of $\operatorname{Hom}_{R}(P, P)$, any component of a split group algebra. We thus have the similar structure theorems for group algebras by considering $P_{1}, P_{2}, \ldots P_{s}$ and $J_{1}, J_{2}, \ldots J_{s}$ in the same time where $P_{i}, i=1,2$, $\cdots s$ are in the definition of a split group algebra $R G$ with

$$
P_{i} \cong \bigoplus \sum_{i=1}^{k_{i}-1} R \oplus J_{i} \text { as in Theorem } 4 .
$$

2. Let us recall the group character of a finitely generated and projective $R G$-module.

Definition 4. Let $M$ be a finitely generated and projective $R G$ module with dual basis $\left\{F_{1}, F_{2}, \cdots F_{u} ; X_{1}, X_{2}, \cdots X_{u}\right\}$. Then the group character $T_{M}: G \rightarrow R$ is defined by $T_{M}(g)=\sum_{i=1}^{u} F_{i}\left(g X_{i}\right)$ for any $g$ in $G([8], \S 2)$.

In this section some properties of group characters will be given. Let $K$ be a field and $K(\varphi)$ be $K\left(\varphi\left(g_{1}\right), \varphi\left(g_{2}\right), \cdots \varphi\left(g_{n}\right)\right)$ where $\varphi$ is a group character for $G=\left\{g_{1}, g_{2}, \cdots g_{n}\right\}$. We know that $K(\varphi)$ is a separable extension over $K$. In the ring case, $R\left[T^{i}\right]$ can be proved as a strongly separable $R$-algebra where $T^{i}$ is a group character for $G$. Finally, we point out the usual orthogonality relations on group 
characters in the ring case.

THEOREM 1. (a) Let $T^{i}$ be $T_{P_{i}}$ where $P_{i}$ is in the definition of a split group algebra $R G$ (see Definition 1 ). Then $T^{i}(g)$ is a constant for all splitting rings $R$ with the same prime ring $R_{0}$ for a given group $G$, where $g$ is in $G$. (b) $T^{i}(g)$ is a sum of $n_{i}^{\text {th }}$-roots of 1 where $g$ is in $G$ and $g^{n_{i}}=1_{G}$, the identity of $G$.

Proof. Since $R$ is a splitting ring for $G, R G \cong \bigoplus \sum_{i=1}^{s} \operatorname{Hom}_{R}\left(P_{i}, P_{i}\right)$. Setting $R^{\prime}=R[\sqrt[m]{1}]$ where $\sqrt[m]{1}$ is a primitive $m^{\text {th }}$-root of 1 and $m$ is the exponent of $G$, we have

$$
\begin{aligned}
R^{\prime} G & \cong R^{\prime} \bigotimes_{R} R G \cong R^{\prime} \otimes_{R}\left(\bigoplus \sum_{i=1}^{s} \operatorname{Hom}_{R}\left(P_{i}, P_{i}\right)\right) \\
& \cong \bigoplus \sum_{i=1}^{s} \operatorname{Hom}_{R^{\prime}}\left(R^{\prime} \bigotimes_{R} P_{i}, R^{\prime} \bigotimes_{R} P_{i}\right) .
\end{aligned}
$$

By Lemma 1 in [8], $R^{\prime}$ is also a splitting ring for $G$. Clearly,

$$
T_{R^{\prime} \otimes_{R} P_{i}}=T^{i} \cdots(1) \text {. }
$$

Next, consider $R^{\prime \prime}=R_{0}[\sqrt[m]{1}]$. It is a splitting ring for $G$ ([8], Th. 2); that is, $R^{\prime \prime} G \cong \bigoplus \sum_{i=1}^{s} \operatorname{Hom}_{R^{\prime \prime}}\left(P_{i}^{\prime \prime}, P_{i}^{\prime \prime}\right)$. We then have

$$
R^{\prime} G \cong R^{\prime} \otimes_{R^{\prime \prime}} R^{\prime \prime} G \cong \bigoplus \sum_{i=1}^{s} \operatorname{Hom}_{R^{\prime}}\left(R^{\prime} \bigotimes_{R^{\prime \prime}} P_{i}^{\prime \prime}, R^{\prime} \bigotimes_{R^{\prime \prime}} P_{i}^{\prime \prime}\right)
$$

Thus $T_{P_{i}^{\prime \prime}}=T_{R^{\prime} \otimes_{R^{\prime}} P_{i}^{\prime \prime}} \cdots(2)$, and for each $i$

$\operatorname{Hom}_{R^{\prime}}\left(R^{\prime} \bigotimes_{R^{\prime \prime}} P_{i}^{\prime \prime}, R^{\prime} \bigotimes_{R^{\prime \prime}} P_{i}^{\prime \prime}\right)=\operatorname{Hom}_{R^{\prime}}\left(R^{\prime} \bigotimes_{R} P_{i}, R^{\prime} \bigotimes_{R} P_{i}\right)$.

The later implies that $R^{\prime} \bigotimes_{R^{\prime \prime}} P_{i}^{\prime \prime} \cong\left(R^{\prime} \bigotimes_{R} P_{i}\right) \bigotimes_{R^{\prime}} J$, where $J$ is in the class group of $R^{\prime}$ ([7], Lemma 9). Consequently,

$$
T_{R^{\prime} \otimes_{R^{\prime}} P_{i}^{\prime \prime}}=T_{R^{\prime} \otimes_{R^{P}} P^{\prime}} \cdots(3) \text {. }
$$

From (1), (2) and (3), $T^{i}=T_{P_{i}^{\prime \prime}}$. But $R^{\prime \prime}$ depends on $R_{0}$ and $G$ only so that $T^{i}$ is a constant for all splitting rings $R$ with the same prime ring $R_{0}$ for a given group $G, i=1,2, \cdots, s$. This proves part (a).

The proof for part (b) divides into two cases. Case 1. Char $(R)$ is equal to $p^{r}$ where $p$ is a prime integer and $r$ is a positive integer. Then the prime ring of $R$ is $Z /\left(p^{r}\right)$ where $Z$ is the set of integers. Let $\sqrt[m]{1}$ be a primitive $m^{\text {th }}$-root of 1 where $m$ is the exponent of $G$. Then $R^{\prime}=Z /\left(p^{r}\right)[\sqrt[m]{1}]$ is a splitting ring for $G$ ([8], Th. 2); that is, $R^{\prime} G \cong \bigoplus \sum_{i=1}^{s} \operatorname{Hom}_{R^{\prime}}\left(P_{i}, P_{i}\right)$. Since $R^{\prime}$ is a local ring (see the proof of Theorem 2 in [8]) and $P_{i}$ is a finitely generated and projective $R^{\prime}$ module for each $i, P_{i}$ is a free $R^{\prime}$-module for each $i$ ([6], Th. 12 in Chapter 9). Therefore $T^{i}(g)$ is a sum of $n_{i}^{\text {th }}$-roots of 1 where $g$ is in 
$G$ and $g^{n_{i}}=1_{G}$, the identity of $G$.

Char $(R)$ is equal to 0 . Then the prime ring of $R$ is $Z(n)$, the quotient ring of $Z$ with respect to the multiplicative closed set $\{n$, $\left.n^{2}, \cdots\right\}$. By the Brauer splitting theorem again, $R^{\prime}=Z(n)[\sqrt[m]{1}]$ is a splitting ring for $G$; that is $R^{\prime} G \cong \bigoplus \sum_{i=1}^{s} \operatorname{Hom}_{R^{\prime}}\left(P_{i}, P_{i}\right)$. Since $R^{\prime}$ is a principal ideal Dedekind domain, $P_{i}$ is a free $R^{\prime}$-module for each $i$ ([4], Exercises 22.5 and 56.6). Therefore $T^{i}(g)$ is a sum of $n_{i}^{\text {th }}$-roots of 1 as in Case 1 .

Theorem 2. Let $R\left[T^{i}\right]$ denote $R\left[T^{i}\left(g_{1}\right), T^{i}\left(g_{2}\right), \cdots\right]$ where $G$ is equal to $\left\{g_{1}, g_{2}, \cdots, g_{n}\right\}$. Then $R\left[T^{i}\right]$ is a strongly separable $R$-algebra for each $i$.

Proof. As in the above theorem, $R$ divides into two cases. Case 1. Char $(R)=0$. Then the prime ring of $R$ is $Z(n)$, the quotient ring of integers with respect to the multiplicative closed set $\left\{n, n^{2}, \cdots\right\}$. We know that the quotient field of $Z(n)\left[T^{i}(g)\right]$ is $Q\left(T^{i}(g)\right)$ for each $g$ in $G$ and the quotient field of $Z(n)[\sqrt[m]{1}]$ is $Q(\sqrt[m]{1})$, where $Q$ is the set of rationals. Because $Z(n)[\sqrt[m]{1}]$ is separable over $Z(n)$ by the Brauer splitting theorem, $Q(\sqrt[m]{1})$ is unramified over $Q$ ([1], Th. 2.5). But $Q\left(T^{i}(g)\right)$ is a subset of $Q(\sqrt[m]{1})$ and contains $Q$; so $Q\left(T^{i}(g)\right)$ is unramified over $Q$ ([9], Proposition 3.2.4). Thus $Z(n)\left[T^{i}(g)\right]$ is separable over $Z(n)$ by Theorem 2.5 in [1] again. This implies that $R \otimes_{Z(n)} Z(n)\left[T^{i}(g)\right]$ is a separable $R$-algebra ([2], Corollary 1.6); so $R\left[T^{i}(g)\right]$, the homomorphic image of $R \otimes_{Z(n)} Z(n)\left[T^{i}(g)\right]$, is also a separable $R$-algebra. On the other hand, because $T^{i}(g)$ is integral over $R, R\left[T^{i}(g)\right]$ is a strongly separable $R$-algebra. Therefore $R\left[T^{i}\right]$ is a strongly separable $R$-algebra.

Case 2. Char $(R)$ is $p^{r}$ for some prime integer $p$ and a positive integer $r$. Then the prime ring of $R$ is $Z /\left(p^{r}\right)$. We know that $Z /\left(p^{r}\right)$ $\left[T^{i}(g)\right]$ is a local ring with the nilpotent maximal ideal $(p) /\left(p^{r}\right)\left[T^{i}(g)\right]$. Also, $Z /\left(p^{r}\right)\left[T^{i}(g)\right]$ is a Noetherian ring such that

$$
(p) /\left(p^{r}\right)\left[T^{i}(g)\right] \cap Z /\left(p^{r}\right)=(p) /\left(p^{r}\right) .
$$

Let $M$ denote $(p) /\left(p^{r}\right)\left[T^{i}(g)\right]$. Then $(p) /\left(p^{r}\right) \cdot\left(Z /\left(p^{r}\right)\left[T^{i}(g)\right]\right)_{M}$ is equal to $M \cdot\left(Z /\left(p^{r}\right)\left[T^{i}(g)\right]\right)_{M}$ for $T^{i}(g)$ is in $M,()_{M}$ is a local ring at $M$.

$$
Z /\left(p^{r}\right)\left[T^{i}(g)\right] /(p) /\left(p^{r}\right)\left[T^{i}(g)\right] \cong Z /(p)\left(T^{i}(g)\right)
$$

is a separable $Z /(p)$ extension. Therefore $Z /\left(p^{r}\right)\left[T^{i}(g)\right]$ is a separable $Z /\left(p^{r}\right)$-algebra $([1], \S 1)$. Then as in Case $1, R\left[T^{i}\right]$ is a strongly separable $R$-algebra by the same arguments. This proves the theorem. 
REMARK. We know that an element $\alpha$ in the separable closure of $R$ is separable means that it satisfies a separable polynomial over $R$. This is also equivalent to that $R[\alpha]$ is a separable $R$-algebra ([5], Lemma 2.7). Then $T^{i}(g)$ is a separable element such that $T^{i}(g)$ is a sum of $n_{i}^{\text {th }}$-roots of 1 . Because these roots satisfy the separable polynomial, $X^{n_{i}}-1=0$, all roots are also separable elements. But it is not true that a sum of separable elements is separable. The following example is due to G. J. Janusz. Let $R$ be $Z(2)$, the quotient ring of $Z$ with respect to the multiplicative closed set $\left\{2,2^{2}, \cdots\right\}, S$ be $R[i]$ where $i^{2}=-1$. Then $S$ is strongly separable over $R$. An element $a+i b$ is a separable element if and only if $(a+i b)-(a-i b)=2 i b$ is invertible in $S$ ([5], Lemma 2.1). Hence the separable elements are of the form $a+i 2^{j}$ where $a$ is in $Z(2)$ and $j=0,1,2, \cdots$. Clearly, $1+i$ and $1+i 2$ are separable elements but $(1+i)+(1+i 2)=(2+i 3)$ is not.

We conclude this section by pointing out the usual orthogonality relations on group characters as in the field case.

THEOREM 3. If $T^{i}=T_{P_{i}}$, for $i=1,2, \cdots, s$, then

$$
\sum_{g} T^{i}(g) T^{j}\left(g^{-1}\right)=n \delta_{i j},
$$

where $n$ is the order of $G$ and $\delta_{i j}$ is the Kronecker delta.

Proof. Let $E_{i}$ be the $i^{\text {th }}$-central primitive idempotent of $R G$,

$$
E_{i}=\sum_{g} \frac{k_{i} T^{i}\left(g^{-1}\right)}{n} g,
$$

where $k_{i}=\operatorname{rank}\left(P_{i}\right)$ ([8], Lemma 5). Taking the characters in both sides, we have the answer.

REMARK. By using the above theorem and standard methods, the other usual orthogonality relations on group characters can be proved (see $\S 31$ in [4]).

The author wishes to thank Professors F. R. DeMeyer and G. J. Janusz for their many valuable suggestions and discussions.

\section{BIBLIOGRAPHY}

1. M. Auslander and D. Buchsbaum, On ramification theory in Noetherian rings, Amer. J. Math. 81 (1959), 749-765.

2. M. Auslander and O. Goldman, The Brauer group of a commutative ring, Trans. Amer. Math. Soc. 97 (1960), 367-409.

3. —, Maximal orders, Trans. Amer. Math. Soc. 97 (1960), 1-24. 
4. C. W. Curtis and I. Reiner, Representation theory of finite groups and associated Algebras, New York, Interscience, 1962.

5. G. J. Janusz, Separable algebra over commutative rings, Trans. Amer. Math. Soc. 122 (1966), 461-479.

6. D. G. Northcott, An introduction to homological algebra, Cambridge University Press, London, 1962.

7. A. Rosenberg and D. Zelinsky, Automorphisms of separable algebras, Pacific J. Math. 2 (1961), 1109-1118.

8. G. Szeto, On the Brauer splitting theorem, Pacific J. Math. 31 (1969), 505-512.

9. E. Weiss, Algebraic number theory, McGraw Hill Book Co., 1963.

Received August 18, 1969.

BRADLEY UNIVERSITY

PEORIA, ILLINOIS 



\section{PACIFIC JOURNAL OF MATHEMATICS}

EDITORS

H. SAMELSON

Stanford University

Stanford, California 94305

RichaRd PIERCE

University of Washington

Seattle, Washington 98105
J. DUGUNDJI

Department of Mathematics

University of Southern California

Los Angeles, California 90007

RICHARD ARENS

University of California

Los Angeles, California 90024

\section{ASSOCIATE EDITORS}

E. F. BECKENBACH

B. H. NeumanN

F. WOLF

K. YOSHIDA

\section{SUPPORTING INSTITUTIONS}

UNIVERSITY OF BRITISH COLUMBIA

CALIFORNIA INSTITUTE OF TECHNOLOGY

UNIVERSITY OF CALIFORNIA

MONTANA STATE UNIVERSITY

UNIVERSITY OF NEVADA

NEW MEXICO STATE UNIVERSITY

OREGON STATE UNIVERSITY

UNIVERSITY OF OREGON

OSAKA UNIVERSITY

UNIVERSITY OF SOUTHERN CALIFORNIA
STANFORD UNIVERSITY

UNIVERSITY OF TOKYO

UNIVERSITY OF UTAH

WASHINGTON STATE UNIVERSITY

UNIVERSITY OF WASHINGTON

AMERICAN MATHEMATICAL SOCIETY CHEVRON RESEARCH CORPORATION TRW SYSTEMS

NAVAL WEAPONS CENTER 


\section{Pacific Journal of Mathematics}

May, 1970

Johan Aarnes, Edward George Effros and Ole A. Nielsen, Locally compact

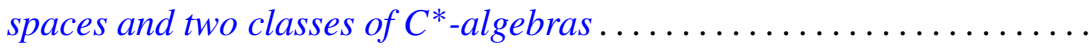

Allan C. Cochran, R. Keown and C. R. Williams, On a class of topological

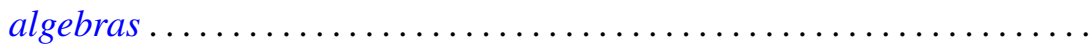

John Dauns, Integral domains that are not embeddable in division rings ....

Robert Jay Daverman, On the number of nonpiercing points in certain

crumpled cubes.....................................

Bryce L. Elkins, Characterization of separable ideals ................

Zbigniew Fiedorowicz, A comparison of two naturally arising uniformities

on a class of pseudo-PM spaces ...........................

Henry Charles Finlayson, Approximation of Wiener integrals of functionals

continuous in the uniform topology ........................

Theodore William Gamelin, Localization of the corona problem ...........

Alfred Gray and Paul Stephen Green, Sphere transitive structures and the

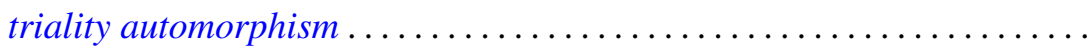

Charles Lemuel Hagopian, On generalized forms of aposyndesis ..........

J. Jakubík, On subgroups of a pseudo lattice ordered group ...............

Cornelius W. Onneweer, On uniform convergence for Walsh-Fourier

series..................................

Stanley Joel Osher, On certain Toeplitz operators in two variables ...

Washek (Vaclav) Frantisek Pfeffer and John Benson Wilbur, On the

measurability of Perron integrable functions............

Frank J. Polansky, On the conformal mapping of variable regions...

Kouei Sekigawa and Shûkichi Tanno, Sufficient conditions for a Riemannian manifold to be locally symmetric ...................

James Wilson Stepp, Locally compact Clifford semigroups ....

Ernest Lester Stitzinger, Frattini subalgebras of a class of solvable Lie

algebras ................................

George Szeto, The group character and split group algebras...

Mark Lawrence Teply, Homological dimension and splitting torsion

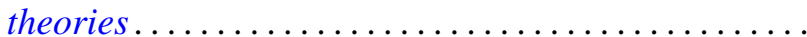

David Bertram Wales, Finite linear groups of degree seven. II ...

Robert Breckenridge Warfield, Jr., An isomorphic refinement theorem for

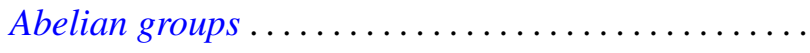

James Edward West, The ambient homeomorphy of an incomplete subspace

of infinite-dimensional Hilbert spaces................

Peter Wilker, Adjoint product and hom functors in general topology ...

Daniel Eliot Wulbert, A note on the characterization of conditional 